\title{
Complexity measures and uncertainty relations of the high-dimensional harmonic and hydrogenic systems
}

\author{
N. Sobrino-Coll, D. Puertas-Centeno, I.V. Toranzo and J. S. Dehesa* \\ Departamento de Física Atómica, Molecular y Nuclear, \\ Universidad de Granada, Granada 18071, Spain and \\ Instituto Carlos I de Física Teórica y Computacional, \\ Universidad de Granada, Granada 18071, Spain
}

\begin{abstract}
In this work we find that not only the Heisenberg-like uncertainty products and the Rényientropy-based uncertainty sum have the same first-order values for all the quantum states of the $D$-dimensional hydrogenic and oscillator-like systems, respectively, in the pseudoclassical $(D \rightarrow \infty)$ limit but a similar phenomenon also happens for both the Fisherinformation-based uncertainty product and the Shannon-entropy-based uncertainty sum, as well as for the Crámer-Rao and Fisher-Shannon complexities. Moreover, we show that the LMC (López-Ruiz-Mancini-Calvet) and LMC-Rényi complexity measures capture the hydrogenic-harmonic difference in the high dimensional limit already at first order.

Keywords: $D$-dimensional quantum physics, $D$-dimensional hydrogenic systems, $D$-dimensional oscillator-like systems, uncertainty relations for high dimensional quantum systems, Complexity of high dimensional quantum systems
\end{abstract}

\section{INTRODUCTION}

The dimensional scaling has been shown to be a powerful tool to describe numerous features of a great variety of (three dimensional) quantum systems and phenomena pertaining to a wide range of fields from atomic and molecular physics [1-5] to general quantum mechanics [ 6 - 8 ], quantum field theory and quantum cosmology [9-13] and quantum information [14-18]. It is often possible to approximate the solution of difficult three-dimensional quantum problems by means of a Taylor series development of similar systems with a non-standard dimensionality (i.e., $D \neq 3$ ) in powers of $1 / D$ and then by using an interpolation or extrapolation procedure.

Indeed, guided by the idea that physics at high dimensions is much simpler, Herschbach et al [1, 2, 4] developed a practical method to investigate the electronic structure of atoms and 
molecules where the dimensional scaling plays the fundamental role. This dimensional scaling method is a very useful strategy to solve the (three-dimensional) finite many-electron problems. This method, which has been mainly used to study the level spectrum and dynamics of Coulomb systems, allows one to solve the associated quantum problem in the pseudoclassical $(D \rightarrow \infty)$ limit, and then, perturbation theory in $1 / D$ is used to have an approximate three-dimensional result, obtaining at times a quantitative accuracy comparable to or better than single-zeta Hartree-Fock calculations $[1-3]$. Thus, the starting and most interesting point here is the $(D \rightarrow \infty)$-limit, tantamount to $h \rightarrow 0$ and/or $m_{e} \rightarrow \infty$ in the kinetic energy, $h$ and $m_{e}$ being the Planck constant and the electron mass, respectively. This limit is not the same as the conventional classical limit obtained by $h \rightarrow 0$ for a fixed dimension [10, 11].

It turns out that the pseudoclassical limit of the finite many-electron problem can be exactly computable in a simple way. The electrons of a finite, high $D$-dimensional atom and molecule are confined to harmonic oscillations about the fixed positions attained in the $(D \rightarrow \infty)$-limit. Indeed, in this limit, the electrons of a many-electron system assume fixed positions relative to the nuclei and each other, in the $D$-scaled space. Moreover, the high- $D$ electronic geometry and energy correspond to the minimum of an exactly known effective potential and can be determined from classical electrostatics for any atom or molecule. Although at first sight, the electrons at rest in fixed locations might seem to violate the uncertainty principle, this is not true because that occurs only in the $D$-scaled space (see, e.g., [4]).

The spatial electron delocalization of the main prototypes of the physics of multidimensional quantum systems, the $D$-dimensional hydrogenic system (i.e., a negatively-charged particle moving in a space of $D$ dimensions around a positively charged core which electromagnetically binds it in its orbit) and the $D$-dimensional harmonic system (i.e., a particle moving under the action of a quadratic potential), has been investigated by means of the Heisenberg-like uncertainty measures which are based on the variance and the moments of the quantum probability of the system with order other than two [19, 22, 24, 25]. Then, uncertainty measures of entropic character [26 34] have been considered; they are much more appropriate because, contrary to the Heisenberg-like ones, they do not depend on any specific point of the system.

Recently these studies have been extended by calculating the dominant term of the Heisenberglike and Rényi-entropy-based uncertainty measures for both the $D$-dimensional hydrogenic and harmonic systems at the quassiclassical border in the two conjugated position and momentum 
spaces [35, 36]. It was found that the Heisenberg-like and Rényi-entropy-based equality-type uncertainty relations for all of the $D$-dimensional harmonic oscillator states in the pseudoclassical $(D \rightarrow \infty)$ limit are the same as the corresponding ones for the hydrogenic systems, despite the so different character of the oscillator and Coulomb potentials. In this work, we investigate whether a similar phenomenon also takes place for both the Fisher-information-based uncertainty product and the Shannon-entropy-based uncertainty sum, as well as for various basic (Crámer-Rao, Fisher-Shannon) and generalized (LMC-Rényi) complexity measures in the two position and momentum spaces.

The structure of the paper is the following. First, in Section I we define and briefly discuss the spreading properties of Heisenberg, entropy and complexity types of a $D$-dimensional probability density which we need for the rest of this work. In Section [II we give the probability densities of the $D$-dimensional hydrogenic and harmonic systems in both position and momentum spaces. In Section IV we calculate and compare not only the position and momentum Heisenberg-like and Rényi-entropy-based uncertainty relations but also the Fisher-information-based uncertainty product and the Shannon-entropy-based uncertainty sum of the $D$-dimensional hydrogenic and harmonic systems. Then, in Section $\mathrm{V}$ we determine and compare the basic and extended complexity measures defined above in both position and momentum spaces. Finally, some conclusions and open problems are given.

\section{ENTROPY AND COMPLEXITY MEASURES OF A $D$-DIMENSIONAL QUANTUM DENSITY}

The physical and chemical properties of these systems are controlled [37] by means of the spatial delocalization or spreading of the single-particle density $\rho(\vec{r}), \vec{r} \in \Delta \subseteq \mathbb{R}^{D}$ defined as

$$
\rho(\vec{r}):=\sum_{\sigma_{1}, \sigma_{2}, \ldots, \sigma_{n}} \int_{\Delta}\left|\Psi\left(\vec{r}, \vec{r}_{2}, \ldots, \vec{r}_{n} ; \sigma_{1}, \sigma_{2}, \ldots, \sigma_{n}\right)\right|^{2} d \vec{r}_{2} \ldots d \vec{r}_{n}
$$

where $\Psi\left(\vec{r}_{1}, \vec{r}_{2}, \ldots, \vec{r}_{n} ; \sigma_{1}, \sigma_{2}, \ldots, \sigma_{n}\right)$ represents the wave function of the $D$-dimensional $n$-particle system, and $\vec{r}=\left(x_{1}, x_{2}, \ldots, x_{D}\right), \sigma_{i} \in\left(-\frac{1}{2}, \frac{1}{2}\right)$, and $\left(r_{i}, \sigma_{i}\right)$ denote the position-spin coordinates of the $i$ th-particle, which is assumed to be normalized and antisymmetrized in the pairs $\left(\vec{r}_{i}, \sigma_{i}\right)$. The power moments or radial expectation values $\left\{\left\langle r^{\alpha}\right\rangle\right\}$ and the entropic moments $\left\{W_{\alpha}[\rho]\right\}$ of the density defined by

$$
\left\langle r^{\alpha}\right\rangle:=\int_{\Delta} r^{\alpha} \rho(\vec{r}) d \vec{r} \quad \text { and } \quad W_{q}[\rho]:=\left\langle\rho^{q-1}\right\rangle=\int_{\Delta}[\rho(\vec{r})]^{q} d \vec{r}
$$


respectively, provide two different (but equivalent) ways to characterize the density $\rho(\vec{r})$ according to the power-moment and entropic-moment problems of Hamburger type [38, 39]. Moreover they describe numerous physical quantities of the system [40-42].

The internal disorder (spatial spreading) of the multidimensional quantum systems can be quantified by entropy and complexity measures (which are closely related to the entropic moments of $\rho(\vec{r}))$ in a much better way than by the Heisenberg-like measures which are linked to the power moments (radial expectation values) of the single-particle probability density $\rho(\vec{r})$, mainly because the latter ones depend on some specific spatial point of the system; so, e.g. the variance $V[\rho]=$ $\left\langle\vec{r}^{2}\right\rangle-\langle\vec{r}\rangle^{2}$ depends on the centroid. The entropy measures (Shannon entropy, disequilibrium, Rényi entropy, Fisher information) not only quantify a spreading facet of the electronic density, but also characterize a great deal of fundamental and/or experimentally accessible energetic quantities of the system as shown by the density functional theory. The Shannon entropy [43, 44] and the disequilibrium (also called informational energy [45]), defined by

$$
S[\rho]:=-\int_{\Delta} \rho(\vec{r}) \ln \rho(\vec{r}) d \vec{r}, \quad \text { and } \quad \mathcal{D}[\rho]:=\int_{\Delta} \rho(\vec{r})^{2} d \vec{r},
$$

measure the total spatial spreading of the electronic charge and the separation with respect to the equiprobability, respectively. The corresponding quantities for the momentum-space probability density $\gamma(\vec{p})$ will be denote by $S[\gamma]$ and $\mathcal{D}[\gamma]$, respectively. Other global spreading facets of the position density $\rho(\vec{r})$ are given by the monoparametric Rényi entropies [46, 47], $R_{q}[\rho]$,

$$
R_{q}[\rho]:=\frac{1}{1-q} \ln \int_{\Delta} \rho(\vec{r})^{q} d \vec{r}, \quad q>0, \quad q \neq 1 .
$$

Note that these quantities include the Shannon entropy, the disequilibrium and the Tsallis entropies $T_{q}[\rho]=\frac{1}{q-1}\left(1-\int_{\mathbb{R}^{3}}[\rho(\vec{r})]^{q}\right)$, since $S[\rho]=\lim _{q \rightarrow 1} R_{q}[\rho], \mathcal{D}[\rho]=\exp \left(-R_{2}[\rho]\right)$ and $T_{q}[\rho]=$ $\frac{1}{1-q}\left[e^{(1-q) R_{q}[\rho]}-1\right]$. The Rényi entropies provide the most relevant canonical class of uncertainty measures [48, 49] and characterize different quantities of the system depending on the $q$-parameter such as the Dirac exchange energy $(q=4 / 3)$, the Thomas-Fermi energy $(q=5 / 3)$, the average density $(q=2), \ldots$ (see e.g., [33, 50, 51]). The corresponding quantities for the momentumspace probability density $\gamma(\vec{p})$ will be denoted by $R_{q}[\gamma]$. The (translationally invariant) Fisher information of the $D$-dimensional density $\rho(\vec{r})$ is defined by

$$
F[\rho]:=\int_{\Delta} \frac{\left|\vec{\nabla}_{D} \rho(\vec{r})\right|^{2}}{\rho(\vec{r})} d \vec{r}
$$

where $\vec{\nabla}_{D}$ denotes the $D$-dimensional gradient of the particle. The corresponding quantity for the momentum-space probability density $\gamma(\vec{p})$ will be denote by $F[\gamma]$. This entropic quantity is 
playing an increasing role in numerous fields [52], particularly for many-electron systems, partially because of its formal resemblance with kinetic [52, 53] and Weiszäcker [54] energies. The Fisher information, contrary to the Rényi, Shannon and Tsallis entropies, is a local measure of spreading of the density $\rho(\vec{r})$ because it is a gradient functional of $\rho(\vec{r})$, so that it is very sensitive to the density fluctuations. Moreover, these entropic measures allow us to characterize and identify some relevant quantum phenomena such as e.g., quantum phase transitions [55], fractality [56], machine learning [57] and the spectral avoided crossings in atoms and molecules [58, 59]. To a great extent this is because the entropic measures satisfy various relevant mathematical properties [60 62$]$ and the position-momentum uncertainty relations [51, 63, 64] (see also [32, 42, 48]) given by

$$
\begin{gathered}
S[\rho]+S[\gamma] \geq D(1+\log \pi) . \\
R_{q}[\rho]+R_{p}[\gamma] \geq D \log \left(p^{\frac{1}{2(p-1)}} q^{\frac{1}{2(q-1)}} \pi\right) \quad \text { with } \frac{1}{p}+\frac{1}{q}=2 . \\
F[\rho] \times F[\gamma] \geq 4 D^{2}
\end{gathered}
$$

for the Shannon, Rényi and Fisher cases, respectively, which improve (i.e., are more stringent) and generalize the Heisenberg position-momentum uncertainty relation $\left\langle r^{2}\right\rangle\left\langle p^{2}\right\rangle \geq \frac{D^{2}}{4}$.

A more complete way to describe the internal order (or disorder) of the multidimensional hydrogenic systems is given by means of the intrinsic complexity measures, which are composed by two information-theoretic measures which quantify simultaneously two facets of the $D$-dimensional quantum density. The two basic complexity measures of this type are the Crámer-Rao measure 65 67] defined by

$$
C_{C R}[\rho]:=F[\rho] \times V[\rho],
$$

and the Fisher-Shannon complexity [68 70$]$ given by

$$
C_{F S}[\rho]:=F[\rho] \times \frac{1}{2 \pi e} e^{\frac{2}{D} S[\rho]} .
$$

where $F[\rho], V[\rho]$ and $S[\rho]$ denote the Fisher information, the variance and the Shannon entropy, respectively, mentioned above.

Recently, a generalized complexity measure has been introduced which extend these two previous measures: the biparametric LMC-Rényi [71 74], defined as

$$
\bar{C}_{\alpha, \beta}[\rho]:=e^{\frac{1}{D}\left(R_{\alpha}[\rho]-R_{\beta}[\rho]\right)}, \quad 0<\alpha<\beta<\infty, \quad \alpha, \beta \neq 1 .
$$


Note that the case $(\alpha \rightarrow 1, \beta=2)$ corresponds to the plain LMC complexity measure [75] $C_{1,2}^{D}[\rho]=$ $\mathcal{D}[\rho] \times e^{S[\rho]}$, which measures the combined balance of the average height of $\rho(\vec{r})$ (by means of the disequilibrium $\mathcal{D}[\rho]=e^{-R_{2}[\rho]}$ ) and its total extent over the density support (by means of the Shannon quantity).

It is worth to remark that all the previous definitions hold in the momentum space where the radial coordinate, $\vec{r}$, is replaced by the momentum one, $\vec{p}$. These three complexity measures are known to be dimensionless, invariant under translation and scaling transformation [76, 77], and universally bounded from below [23, $50,61,78]$ as

$$
C_{C R}[\rho] \geq D^{2}, \quad C_{F S}[\rho] \geq D, \quad \text { and } \quad \bar{C}_{\alpha, \beta}[\rho] \geq 1 \quad \text { if } \quad \alpha<\beta
$$

for $D$-dimensional probability densities. The corresponding complexity measures for the momentum-space probability density $\gamma(\vec{p})$ will be denoted by $C_{C R}[\gamma], C_{F S}[\gamma]$ and $\bar{C}_{\alpha, \beta}[\gamma]$, respectively.

\section{HYDROGENIC AND HARMONIC DENSITIES IN POSITION AND MOMENTUM SPACES}

In this section we gather the known probability densities of the $D$-dimensional $(D>1)$ hydrogenic and harmonic systems in position and momentum spaces [26], which are the basic variables to determine the corresponding uncertainty measures in the rest of the paper. Atomic units are used throughout the paper.

\section{A. D-dimensional hydrogenic probability density}

The stationary bound states $(n, l,\{\mu\})$ of the $D$-dimensional $(D>1)$ hydrogenic system (i.e. a particle subject to a central potential of the form $\mathcal{V}_{H}(r)=-\frac{Z}{r}$, being $Z$ the nuclear charge) are known (see e.g., [26]) to have the energies $E_{\eta}=-\frac{Z^{2}}{\eta^{2}}$, (with the grand quantum number $\eta=n+\frac{D-3}{2}$, and $\left.n=1,2, \ldots\right)$ and the associated probability density is given by

$$
\rho_{n, l,\{\mu\}}^{(H)}(\vec{r})=\frac{\Lambda^{-D}}{2 \eta} \frac{\omega_{2 L+1}(\tilde{r})}{\tilde{r}^{D-2}}\left[\tilde{\mathcal{L}}_{\eta-L-1}^{(2 L+1)}(\tilde{r})\right]^{2}\left|\mathcal{Y}_{l,\{\mu\}}\left(\Omega_{D-1}\right)\right|^{2},
$$

in position space, where the position vector $\vec{r}=\left(r, \theta_{1}, \theta_{2}, \ldots, \theta_{D-1}\right)$ in polar hyperspherical coordinates, $(n, l,\{\mu\})=\left(n, l \equiv \mu_{1}, \ldots, \mu_{D-1}\right)$ are the corresponding hyperquantum numbers with values $\left\{l=0,1,2, \ldots, n-1 ; \quad l \geq \mu_{2} \geq \ldots \geq \mu_{D-1} \equiv|m| \geq 0\right\}$, and

$$
L \equiv l+\frac{D-3}{2}, \quad \tilde{r} \equiv \frac{r}{\lambda} \quad \text { and } \quad \Lambda \equiv \frac{\eta}{2 Z}
$$


The symbol $\tilde{\mathcal{L}}_{k}^{(\alpha)}(x)$ denotes the orthonormal Laguerre polynomials of degree $k$ and parameter $\alpha=2 l+D-1$ with respect to the weight function $\omega_{\alpha}(x)=x^{\alpha} e^{-x}$ on the interval $[0, \infty)$. The angular part, $\mathcal{Y}_{l,\{\mu\}}\left(\Omega_{D-1}\right)$, denotes the hyperspherical harmonics [7, 26] given by

$$
\mathcal{Y}_{l,\{\mu\}}\left(\Omega_{D-1}\right)=\frac{1}{\sqrt{2 \pi}} e^{i m \phi} \prod_{j=1}^{D-2} \tilde{C}_{\mu_{j}-\mu_{j+1}}^{\left(\alpha_{j}+\mu_{j+1}\right)}\left(\cos \theta_{j}\right)\left(\sin \theta_{j}\right)^{\mu_{j+1}},
$$

with $\alpha_{j}=\frac{1}{2}(D-j-1)$ and $\tilde{C}_{k}^{(\alpha)}(x)$ denotes the Gegenbauer polynomials of degree $k$ and parameter $\alpha$ orthonormal with respect to the weight function $\omega_{\alpha}^{*}(x)=\left(1-x^{2}\right)^{\alpha-\frac{1}{2}}$ on the interval [-1, 1] [79] .

Likewise, the probability density of these systems in the $D$-dimensional momentum space [26] is given as

$$
\gamma_{n, l,\{\mu\}}^{(H)}(\vec{p})=\left(\frac{\eta}{Z}\right)^{D}(1+y)^{3}\left(\frac{1+y}{1-y}\right)^{\frac{D-2}{2}} \omega_{L+1}^{*}(y)\left[\tilde{C}_{\eta-L-1}^{(L+1)}(y)\right]^{2}\left[\mathcal{Y}_{l,\{\mu\}}\left(\Omega_{D-1}\right)\right]^{2}
$$

with $\vec{p}=\left(p, \theta_{1}, \ldots, \theta_{D-1}\right)$ and the notation

$$
y \equiv \frac{1-\eta^{2} \tilde{p}^{2}}{1+\eta^{2} \tilde{p}^{2}}, \quad \text { and } \quad \tilde{p}=\frac{p}{Z} .
$$

\section{B. D-dimensional harmonic probability density}

The stationary bound states $(n, l,\{\mu\})$ of the $D$-dimensional harmonic system (i.e. a particle subject to a central potential of the oscillator form $\mathcal{V}_{O}(r)=\frac{1}{2} \lambda^{2} r^{2}$ ) are known (see e.g., [26]) to have the energies $E=\lambda\left(2 n+l+\frac{D}{2}\right)$ (with $n=0,1,2, \ldots$ and $\left.l=0,1,2, \ldots\right)$ and the associated probability density is given by

$$
\rho_{n, l,\{\mu\}}^{(O)}(\vec{r})=\rho_{n, l}^{(O)}(r)\left|\mathcal{Y}_{l,\{\mu\}}\left(\Omega_{D-1}\right)\right|^{2},
$$

in position space, where $\rho_{n, l}(r)$ denotes the radial part of the density defined as

$$
\rho_{n, l}^{(O)}(r)=\frac{2 n ! \lambda^{l+\frac{D}{2}}}{\Gamma\left(n+l+\frac{D}{2}\right)} e^{-\lambda r^{2}} r^{2 l}\left[\mathcal{L}_{n}^{\left(l+\frac{D}{2}-1\right)}\left(\lambda r^{2}\right)\right]^{2},
$$

with $\left\{n=1,2,3, \ldots ; \quad l=0,1,2, \ldots ; \quad l \geq \mu_{2} \geq \ldots \geq \mu_{D-1} \equiv|m| \geq 0\right\}$. The symbol $\mathcal{L}_{n}^{(\alpha)}(x)$ denotes the orthogonal Laguerre polynomials [79] with respect to the weight $\omega_{\alpha}(x)=x^{\alpha} e^{-x}, \alpha=$ $l+\frac{D}{2}-1$, on the interval $[0, \infty)$.

On the other hand, in the conjugated space, the Fourier transform provides the following expression

$$
\gamma_{n, l,\{\mu\}}^{(O)}(\vec{p})=\left|\tilde{\Psi}_{n, l,\{\mu\}}^{(O)}(\vec{p})\right|^{2}=\lambda^{-D} \rho_{n, l,\{\mu\}}^{(O)}\left(\frac{\vec{p}}{\lambda}\right)
$$


for the momentum probability density of the $D$-dimensional harmonic stationary state with the hyperquantum numbers $(n, l,\{\mu\})$.

\section{UNCERTAINTY RELATIONS FOR HYDROGENIC AND HARMONIC SYSTEMS AT HIGH $D$}

In this section we first realize that the Heisenberg-like uncertainty products of the $D$-dimensional hydrogenic and harmonic systems have the same value at high $D$. Then we prove that the same phenomenon occurs for the other mathematical realizations of the position-momentum uncertainty principle based on uncertainty measures of entropic type, such as the Fisher information and the Rényi and Shannon entropies.

\section{A. Heisenberg-like uncertainty relation}

For all stationary bound states $(n, l,\{\mu\})$ of both hydrogenic and harmonic systems we have that the position-space variance $V\left[\rho_{n, l,\{\mu\}}\right]=\left\langle\vec{r}^{2}\right\rangle-\langle\vec{r}\rangle^{2}=\left\langle r^{2}\right\rangle$, since $\langle\vec{r}\rangle=0$ for any central potential. As well, in momentum space we have that the corresponding variance is $V\left[\gamma_{n, l,\{\mu\}}\right]=\left\langle p^{2}\right\rangle$. It is

known [33] that $\left\langle r^{2}\right\rangle_{H}=\frac{\eta^{2}}{2 Z^{2}}\left[5 \eta^{2}+1-3 L(L+1)\right]$ and $\left\langle p^{2}\right\rangle_{H}=\frac{Z^{2}}{\eta^{2}}$ for hydrogenic systems, so that the hydrogenic Heisenberg uncertainty product is given as

$$
\left\langle r^{2}\right\rangle_{H}\left\langle p^{2}\right\rangle_{H}=\frac{D^{2}}{4}\left\{1+\frac{1}{D}(10 n-6 l-9)+\frac{1}{D^{2}}[10 n(n-3)-6 l(l-2)+20]\right\} .
$$

Similarly, since $\left\langle r^{2}\right\rangle_{O}=\lambda^{-1}\left(2 n+l+\frac{D}{2}\right)$ and $\left\langle p^{2}\right\rangle_{O}=\lambda\left(2 n+l+\frac{D}{2}\right)$ for harmonic (i.e., oscillatorlike) systems, we have the following harmonic Heisenberg uncertainty product

$$
\left\langle r^{2}\right\rangle_{O}\left\langle p^{2}\right\rangle_{O}=\left(2 n+l+\frac{D}{2}\right)^{2}=\frac{D^{2}}{4}\left\{1+\frac{1}{D}(8 n+4 l)+\frac{1}{D^{2}}\left[4(2 n+l)^{2}\right]\right\} .
$$

Then, it is clear that the Heisenberg uncertainty product has the value

$$
\left\langle r^{2}\right\rangle_{i}\left\langle p^{2}\right\rangle_{i}=\frac{D^{2}}{4}\left(1+\mathcal{O}\left(\frac{1}{D}\right)\right)
$$

(with $i=H, O$ ) for both $D$-dimensional hydrogenic and harmonic systems. Moreover, it is possible to find that the generalized Heisenberg (or Heisenberg-like) uncertainty product for these two classes of systems is given [24] by

$$
\left\langle r^{\alpha}\right\rangle_{i}\left\langle p^{\alpha}\right\rangle_{i}=\left(\frac{D}{2}\right)^{\alpha}\left(1+\mathcal{O}\left(\frac{1}{D}\right)\right),
$$


(with $i=H, O)$ which holds for $\alpha \in(-D-2 l, D+2 l+2)$ in the hydrogenic case and for $\alpha>-D-2 l$ in the oscillator case. To obtain this result we have taken into account that the position radial expectation value of the hydrogenic system is

$$
\begin{aligned}
\left\langle r^{\alpha}\right\rangle_{H} & =\int r^{\alpha} \rho_{n, l,\{\mu\}}^{(H)}(\vec{r}) d \vec{r} \\
& =\frac{1}{2 \eta}\left(\frac{\eta}{2 Z}\right)^{\alpha} \int_{0}^{\infty} \omega_{2 l+D-2}(t)\left[\tilde{\mathcal{L}}_{n-l-1}^{(2 l+D-2)}(t)\right]^{2} \tilde{r}^{\alpha+1} d t \\
& =\left(\frac{D^{2}}{4 Z}\right)^{\alpha}\left(1+\mathcal{O}\left(\frac{1}{D}\right)\right),
\end{aligned}
$$

(which holds for $\alpha>-D-2 l$ ), and the corresponding momentum radial expectation value is

$$
\begin{aligned}
\left\langle p^{\alpha}\right\rangle_{H} & =\int p^{\alpha} \gamma_{n, l,\{\mu\}}^{(H)}(\vec{p}) d \vec{p} \\
& =\left(\frac{Z}{\eta}\right)^{\alpha} \int_{-1}^{1} \omega_{\nu}^{*}(t)\left[\tilde{\mathcal{C}}_{k}^{(\nu)}(t)\right]^{2}(1-t)^{\frac{\alpha}{2}}(1+t)^{1-\frac{\alpha}{2}} d t \\
& =\left(\frac{2 Z}{D}\right)^{\alpha}\left(1+\mathcal{O}\left(\frac{1}{D}\right)\right),
\end{aligned}
$$

which holds for $\alpha \in(-D-2 l, D+2 l+2)$. Here the notations $k=\eta+L+1=n-l-1$ and $\nu=L+1=l+(D-1) / 2$ have been used. For the third equality of Eqs. (24) and (25) we have considered that the position and momentum expectation values can be expressed [33, 80, 81] in terms of hypergeometric functions ${ }_{3} F_{2}(1)$ and ${ }_{5} F_{4}(1)$, respectively, and then we have used the asymptotics of these functions at high $D$; see [24] for further details.

Similarly, the corresponding radial expectation values of the $D$-dimensional harmonic system are given by

$$
\begin{aligned}
\left\langle r^{\alpha}\right\rangle & =\int r^{\alpha} \rho_{n, l,\{\mu\}}^{(O)}(\vec{r}) d \vec{r} \\
& =\frac{n ! \lambda^{-\alpha / 2}}{\Gamma(n+l+D / 2)} \int_{0}^{\infty} x^{l+\frac{D+\alpha}{2}-1} e^{-x}\left[\mathcal{L}_{n}^{\left(l+\frac{D}{2}-1\right)}(x)\right]^{2} d x \\
& =\left(\frac{D}{2 \lambda}\right)^{\frac{\alpha}{2}}\left(1+\mathcal{O}\left(\frac{1}{D}\right)\right)
\end{aligned}
$$

(valid for $\alpha>-D-2 l$ ) in position space, and

$$
\begin{aligned}
\left\langle p^{\alpha}\right\rangle & =\int p^{\alpha} \gamma_{n, l,\{\mu\}}^{(O)}(\vec{p}) d \vec{p} \\
& =\frac{n ! \lambda^{\alpha / 2}}{\Gamma(n+l+D / 2)} \int_{0}^{\infty} u^{l+\frac{D+\alpha}{2}-1} e^{-u}\left[\mathcal{L}_{n}^{\left(l+\frac{D}{2}-1\right)}(u)\right]^{2} d u \\
& =\left(\frac{\lambda D}{2}\right)^{\frac{\alpha}{2}}\left(1+\mathcal{O}\left(\frac{1}{D}\right)\right)
\end{aligned}
$$

(valid for $\alpha>-D-2 l$ ) in momentum space. In writing the third equality of Eqs. (26) and (27) we first realize that both quantities are entropic functionals of Laguerre polynomials, and then we use 
the recently found asymptotics for these functionals at large parameters [82]; see [35] for further details. Now, note that the multiplication of the hydrogenic expressions (24) and (25), and the harmonic expressions (26) and (27) gives rise to the wanted result (23).

From Eqs. (20) and (21) we remark that the Heisenberg-like products for both $D$-dimensional hydrogenic and harmonic states do not depend on the magnetic hyperquantum numbers of the states. Moreover, from Eqs. (22) and (23) at first order of the pseudoclassical $(D \rightarrow \infty)$ limit both Heisenberg-like products are equal to the saturation value of the position-momentum uncertainty relation (i.e., $\left\langle r^{2}\right\rangle\left\langle p^{2}\right\rangle \geq \frac{D^{2}}{4}$; see also [20-23]) which holds for general $D$-dimensional quan tum systems. Consequently, the first-order Heisenberg-like products at the pseudoclassical border do not capture the qualitatively different character of the Coulomb and quadratic forces which characterize the hydrogenic and harmonic systems, respectively.

\section{B. Fisher-information-based uncertainty relation}

The Fisher information for an arbitrary bound state $(n, l,\{\mu\})$ of a $D$-dimensional single-particle system subject to a central potential $\mathcal{V}(r)$, with the probability densities $\rho \equiv \rho_{n, l,\{\mu\}}(\vec{r})$ and $\gamma \equiv$ $\gamma_{n, l,\{\mu\}}(\vec{p})$ in the two conjugated spaces, can be expressed [83, 84] as

$$
F[\rho]=4\left\langle p^{2}\right\rangle-2|m|(2 l+D-2)\left\langle r^{-2}\right\rangle
$$

in position space, and as

$$
F[\gamma]=4\left\langle r^{2}\right\rangle-2|m|(2 l+D-2)\left\langle p^{-2}\right\rangle
$$

in momentum space, in terms of the pairs of radial expectation values $\left(\left\langle p^{2}\right\rangle,\left\langle r^{-2}\right\rangle\right)$ and $\left(\left\langle r^{2}\right\rangle,\left\langle p^{-2}\right\rangle\right)$, respectively.

Let us first apply these expressions to the hydrogenic system. Since $\left\langle p^{2}\right\rangle_{H}=\frac{Z^{2}}{\eta^{2}},\left\langle r^{-2}\right\rangle_{H}=$ $\frac{2 Z^{2}}{\eta^{3}} \frac{1}{2 L+1}$, and $\left\langle p^{-2}\right\rangle_{H}=\frac{\eta^{2}}{Z^{2}} \frac{8 \eta-3(2 L+1)}{2 L+1}$, one has [33] the following values for the position and momentum Fisher informations

$$
\begin{aligned}
& F\left[\rho^{(H)}\right]=\int \frac{\left|\vec{\nabla}_{D} \rho^{H}(\vec{r})\right|^{2}}{\rho(\vec{r})} d \vec{r}=\frac{4 Z^{2}}{\eta^{3}}[\eta-|m|], \\
& F\left[\gamma^{(H)}\right]=\int \frac{\left|\vec{\nabla}_{D} \gamma^{H}(\vec{p})\right|^{2}}{\gamma(\vec{p})} d \vec{p}=\frac{2 \eta^{2}}{Z^{2}}\left[5 \eta^{2}-3 L(L+1)-|m|(8 \eta-6 L-3)+1\right],
\end{aligned}
$$

in position and momentum spaces, respectively. Then, at high $D$ these expressions simplify as

$$
F\left[\rho^{(H)}\right]=\frac{16 Z^{2}}{D^{2}}\left(1+\mathcal{O}\left(\frac{1}{D}\right)\right)
$$




$$
F\left[\gamma^{(H)}\right]=\frac{D^{4}}{4 Z^{2}}\left(1+\mathcal{O}\left(\frac{1}{D}\right)\right)
$$

for the position and momentum Fisher informations at the pseudoclassical limit.

Working similarly for the $D$-dimensional harmonic system one finds from Eqs. (28) and (29) the following values

$$
\begin{aligned}
& F\left[\rho^{(O)}\right]=\int \frac{\left|\vec{\nabla}_{D} \rho^{O}(\vec{r})\right|^{2}}{\rho(\vec{r})} d \vec{r}=4\left(\eta+|m|+\frac{3}{2}\right) \lambda, \\
& F\left[\gamma^{(O)}\right]=\int \frac{\left|\vec{\nabla}_{D} \gamma^{O}(\vec{r})\right|^{2}}{\rho(\vec{r})} d \vec{r}=4\left(\eta-|m|+\frac{3}{2}\right) \lambda^{-1},
\end{aligned}
$$

for the Fisher information in position and momentum spaces, respectively. Note that both quantities depend on two hyperquantum numbers, $n$ and $m \equiv \mu_{D-1}$ only. Then, for fixed $n$ and $m$ the asymptotics of the Fisher information at high $D$ turns out to be

$$
\begin{aligned}
& F\left[\rho^{(O)}\right]=2 \lambda D\left(1+\mathcal{O}\left(\frac{1}{D}\right)\right), \\
& F\left[\gamma^{(O)}\right]=\frac{2 D}{\lambda}\left(1+\mathcal{O}\left(\frac{1}{D}\right)\right),
\end{aligned}
$$

in position and momentum spaces, respectively.

Finally, the multiplication of the hydrogenic expressions (32) and (33) and the harmonic expressions (36) and (37) gives rise to the following Fisher-information-based uncertainty product

$$
F\left[\rho^{(i)}\right] \times F\left[\gamma^{(i)}\right]=4 D^{2}\left(1+\mathcal{O}\left(\frac{1}{D}\right)\right)
$$

(with $i=H, O$ ) for both $D$-dimensional hydrogenic and harmonic systems at high $D$. Note that this Fisher-information-based uncertainty product (i) fulfils the Fisher-information-based uncertainty relation (8) for general systems, and (ii) cannot disentangle between the Coulomb and oscillator-like forces at the pseudoclassical edge.

\section{Rényi-information-based uncertainty relation}

The Rényi entropy for a generic $(n, l,\{\mu\})$-state of a $D$-dimensional hydrogenic system is given, according to Eqs. (4), (13) and (16), by

$$
R_{q}\left[\rho_{n, l,\{\mu\}}^{(H)}\right]:=\frac{1}{1-q} \log \left(\int_{\Delta}\left[\rho_{n, l,\{\mu\}}^{(H)}(\vec{r})\right]^{q} d \vec{r}\right)
$$

and

$$
R_{q}\left[\gamma_{n, l,\{\mu\}}^{(H)}\right]:=\frac{1}{1-q} \log \left(\int_{\Delta}\left[\gamma_{n, l,\{\mu\}}^{(H)}(\vec{p})\right]^{q} d \vec{p}\right)
$$


in position and momentum spaces, respectively. These two quantities can be decomposed into two radial and angular parts which can be expressed in terms of entropic functionals of Laguerre and Gegenbauer polynomials, respectively. Then, we can use the 2017-dated asymptotics of these functionals for large values of the polynomial parameters [82] to find the following values for the position and momentum Rényi entropies of the high D-dimensional hydrogenic system [36]:

$$
\begin{aligned}
R_{q}\left[\rho_{n, l,\{\mu\}}^{(H)}\right]= & \frac{3}{2} D \log \left(\frac{D}{2}\right)+D \log \left(\frac{q^{\frac{1}{q-1}}}{Z} \sqrt{\frac{\pi}{e}}\right)+\frac{q(n-l-1)}{1-q} \log D \\
& +\mathcal{O}(1), \\
R_{q}\left[\gamma_{n, l,\{\mu\}}^{(H)}\right]= & -\frac{3}{2} D \log \left(\frac{D}{2}\right)+D \log \left(\frac{Z \sqrt{e \pi}}{\tilde{q}^{\frac{1}{q-1}}}\right)+\frac{q(n-l-1)}{1-q} \log D \\
& +\mathcal{O}(1),
\end{aligned}
$$

respectively, where $\tilde{q}=\left(\frac{(2 q-1)^{2 q-1}}{q^{2 q}}\right)^{\frac{1}{2}}$. Note that the Coulomb-strength manifestation appears in the second term and the dependence on the quantum numbers $(n, l)$ do not appear up to the third term in both position and momentum Rényi entropies.

Similar operations in the $D$-dimensional harmonic (oscillator-like) system have led us to the following values for the position and momentum Rényi entropies of this system at high $D$ :

$$
\begin{aligned}
R_{q}\left[\rho_{n, l,\{\mu\}}^{(O)}\right]= & \frac{1}{1-q} \log \left(\int_{\Delta}\left[\rho_{n, l,\{\mu\}}^{O}(\vec{r})\right]^{q} d \vec{r}\right)=\frac{D}{2} \log \left(\frac{q^{\frac{1}{q-1}} \pi}{\lambda}\right)+\frac{q n}{1-q} \log D \\
& +\mathcal{O}(1) \\
R_{q}\left[\gamma_{n, l,\{\mu\}}^{(O)}\right]= & \frac{1}{1-q} \log \left(\int_{\Delta}\left[\gamma_{n, l,\{\mu\}}^{O}(\vec{p})\right]^{q} d \vec{p}\right)=\frac{D}{2} \log \left(q^{\frac{1}{q-1}} \pi \lambda\right)+\frac{q n}{1-q} \log D \\
& +\mathcal{O}(1),
\end{aligned}
$$

in position and momentum spaces, respectively. Note that the oscillator-strength manifestation appears in the dominant term and the dependence on the quantum numbers $(n, l)$ does not appear up to the second term in both position and momentum Rényi entropies.

Finally, from the hydrogenic expressions (41) and (42) and the harmonic expressions (43) and (44) we can determine the position-momentum uncertainty Rényi-entropy-based sum for both highdimensional hydrogenic and harmonic systems, obtaining the values

$$
R_{p}\left[\rho_{n, l,\{\mu\}}^{(i)}\right]+R_{q}\left[\gamma_{n, l\{\mu\}}^{(i)}\right] \sim D \log \left(\pi p^{\frac{1}{2(p-1)}} q^{\frac{1}{2(q-1)}}\right), \quad \text { if } \quad D>>1
$$

(with $i=H, O$ ) where $\frac{1}{p}+\frac{1}{q}=2$ (i.e., $\frac{p}{p-1}+\frac{q}{q-1}=0$ or $q=\frac{p}{2 p-1}$ ), which saturates the uncertainty Rényi-entropy-based relation (7) in both hydrogenic and harmonic cases. The symbol $A \sim B \Leftrightarrow$ $\frac{A}{B} \rightarrow 1$. 
Note that in the limiting case with $q \rightarrow 1$ and $p \rightarrow 1$, this expression gives the following dominant term of the position-momentum Shannon-entropy-based uncertainty sum

$$
S\left[\rho_{n, l,\{\mu\}}^{(i)}\right]+S\left[\gamma_{n, l,\{\mu\}}^{(i)}\right] \sim D \log (e \pi), \quad \text { if } \quad D>>1,
$$

(with $i=H, O$ ) for both high-dimensional hydrogenic and harmonic systems, which saturates the uncertainty Shannon-entropy-based relation (6) in both hydrogenic and harmonic cases. In doing this, we have taken into account the definition (3) of the Shannon entropy for the position and momentum probability densities of these systems which are given by Eqs. (13) and (16), (17) and (19), respectively.

Finally, from expressions (45) and (46) we observe that the uncertainty Rényi-entropy-based and Shannon-entropy-based sums cannot unravel either between the Coulomb and oscillator-like forces at the pseudoclassical border. In conclusion, neither the Heisenberg-like and the Fisherinformation-based uncertainty products nor the entropic uncertainty sums are able to capture any force manifestation at the pseudoclassical border, at least at first order.

\section{COMPLEXITIES OF HYDROGENIC AND HARMONIC SYSTEMS AT HIGH $D$}

In this section we will determine the leading term of the position and momentum complexity measures of Crámer-Rao, Fisher-Shannon and LMC-Rényi types for high-dimensional systems of hydrogenic and harmonic character.

The Crámer-Rao complexity (9) of the $D$-dimensional hydrogenic and harmonic states characterized by the hyperquantum numbers $(n, l,\{\mu\})$ are given by

$$
\begin{aligned}
& C_{C R}\left[\rho^{(i)}\right]=F\left[\rho^{(i)}\right] \times V\left[\rho^{(i)}\right]=D^{2}+\mathcal{O}(D), \\
& C_{C R}\left[\gamma^{(i)}\right]=F\left[\gamma^{(i)}\right] \times V\left[\gamma^{(i)}\right]=D^{2}+\mathcal{O}(D),
\end{aligned}
$$

(for $i=H, O$ ) in position and momentum spaces, respectively. To obtain these high-dimensional complexity values we have used (a) the expressions (32), (33), (36) and (37) for the position and momentum Fisher information of the hydrogenic and harmonic systems, respectively, and (b) the following values for the involved variances

$$
\begin{aligned}
V^{(H)}\left[\rho_{n, l,\{\mu\}}\right] & \sim\left(\frac{D^{2}}{4 Z}\right)^{2} ; \quad V^{(H)}\left[\gamma_{n, l,\{\mu\}}\right] \sim\left(\frac{2 Z}{D}\right)^{2}, \quad \text { if } D>>1, \\
V^{(O)}\left[\rho_{n, l,\{\mu\}}\right] & \sim \frac{D}{2 \lambda} ; \quad V^{(O)}\left[\gamma_{n, l,\{\mu\}}\right] \sim \frac{\lambda D}{2}, \quad \text { if } \quad D>>1,
\end{aligned}
$$

which were derived from the general related expressions (24), (25), (26) and (27) found in the previous section. 
From Eqs. (47) and (48) we realize that these Crámer-Rao values attain the universal lower bound $D^{2}$ in both hydrogenic and harmonic cases at the high-dimensional limit. This means that the Crámer-Rao complexity cannot untangle between Coulomb and oscillator-like systems at the pseudoclassical edge.

The Fisher-Shannon complexities (10) of the $D$-dimensional hydrogenic and harmonic states characterized by the hyperquantum numbers $(n, l,\{\mu\})$ are given by

$$
\begin{aligned}
& C_{F S}\left[\rho^{(i)}\right]=F\left[\rho^{(i)}\right] \times \frac{1}{2 \pi e} e^{\frac{2}{D} S\left[\rho^{(i)}\right]}=D+\mathcal{O}(1), \\
& C_{F S}\left[\gamma^{(i)}\right]=F\left[\gamma^{(i)}\right] \times \frac{1}{2 \pi e} e^{\frac{2}{D} S\left[\gamma^{(i)}\right]}=D+\mathcal{O}(1),
\end{aligned}
$$

(for $i=H, O$ ) in position and momentum space, respectively. These high-dimensional complexity values have been obtained by means of (a) the expressions (32), (33), (36) and (37) for the position and momentum Fisher information of the hydrogenic and harmonic systems, respectively, and (b) the following values for the involved Shannon entropies [36]

$$
\begin{aligned}
& S\left[\rho^{(H)}\right]=\frac{3}{2} D \log \left(\frac{D}{2}\right)+D \log \left(\frac{\sqrt{e \pi}}{Z}\right)+\mathcal{O}(\log D), \\
& S\left[\gamma^{(H)}\right]=-\frac{3}{2} D \log \left(\frac{D}{2}\right)+D \log (Z \sqrt{e \pi})+\mathcal{O}(\log D),
\end{aligned}
$$

and

$$
\begin{aligned}
S^{(O)}\left[\rho_{n, l,\{\mu\}}\right] & \sim \frac{D}{2} \log \left(\frac{e \pi}{\lambda}\right) \\
S^{(O)}\left[\gamma_{n, l,\{\mu\}}\right] & \sim \frac{D}{2} \log (e \pi \lambda),
\end{aligned}
$$

for hydrogenic and harmonic systems in position and momentum spaces, respectively.

Finally we observe from Eqs. (51) and (52) that these Fisher-Shannon values reach the universal lower bound $D$ in both hydrogenic and harmonic cases at the high-dimensional limit. This means that the Fisher-Shannon complexity cannot disentangle either between Coulomb and oscillator-like systems at the pseudoclassical border.

The LMC-Rényi complexities (11) of the D-dimensional hydrogenic and harmonic states characterized by the hyperquantum numbers $(n, l,\{\mu\})$ are given by

$$
\begin{aligned}
& \bar{C}_{\alpha, \beta}\left[\rho^{(i)}\right]=e^{\frac{1}{D}\left(R_{\alpha}\left[\rho^{(i)}\right]-R_{\beta}\left[\rho^{(i)}\right]\right)}, \\
& \bar{C}_{\alpha, \beta}\left[\gamma^{(i)}\right]=e^{\frac{1}{D}\left(R_{\alpha}\left[\gamma^{(i)}\right]-R_{\beta}\left[\gamma^{(i)}\right]\right)},
\end{aligned}
$$

(with $i=H, O$ ) in position and momentum space, respectively, for $0<\alpha<\beta<\infty$ and $\alpha, \beta \neq 1$. 
The determination of the these quantities at high dimensions is straightforward but a bit tedious from the high-dimensional expressions of the corresponding position and momentum Rényi entropies which were derived in the previous section. Indeed, from the expressions (41) and (43) in position space and the expressions (42) and (44) in momentum space we have obtained the following dominant term for the hydrogenic and harmonic LMC-Rényi complexity values

$$
\begin{aligned}
& \left.\bar{C}_{\alpha, \beta}\left[\rho_{n, l,\{\mu\}}^{(H)}\right] \sim\left(\frac{\alpha^{\frac{1}{\alpha-1}}}{\beta^{\frac{1}{\beta-1}}}\right)^{\frac{1}{H^{\frac{1}{\alpha-1}}}}\right)^{\frac{1}{2}} \\
& \bar{C}_{\alpha, \beta}\left[\rho_{n, l,\{\mu\}}^{(O)}\right] \sim\left(\frac{1}{\beta-1}\right)^{(O)}
\end{aligned}
$$

in position space, and

$$
\begin{aligned}
& \bar{C}_{\alpha, \beta}\left[\gamma_{n, l,\{\mu\}}^{(H)}\right] \sim\left(\frac{(2 \alpha-1)^{\frac{2 \alpha-1}{2(1-\alpha)}}}{(2 \beta-1)^{\frac{2 \beta-1}{2(1-\beta)}}}\right)\left(\frac{\alpha^{\frac{\alpha}{\alpha-1}}}{\beta^{\frac{\beta}{\beta-1}}}\right) \\
& \bar{C}_{\alpha, \beta}\left[\gamma_{n, l,\{\mu\}}^{(O)}\right] \sim\left(\frac{\alpha^{\frac{1}{\alpha-1}}}{\beta^{\frac{1}{\beta-1}}}\right)^{\frac{1}{2}},
\end{aligned}
$$

in momentum space in the pseudoclassical limit. We realize by looking at the position expressions (59) and (60) and the momentum expressions (61) and (62) that, contrary to the uncertainty relations and entropic and complexity quantities previously considered in this work, both the position and momentum LMC-Rényi complexities are able to disentangle between the hydrogenic and harmonic systems at first order in the high dimensional limit. Moreover, at this dominant order these two position and momentum generalized measures of complexity do not depend on any hyperquantum numbers which characterize the system's state.

\section{CONCLUSIONS}

In this work the $D$-dimensional hydrogenic and harmonic (oscillator-like) systems, which are the main prototypes in the multidimensional quantum physics, have been investigated at the pseudoclassical limit of high dimensions in both position and momentum spaces. We have used various spreading quantities of their associated probability densities, such as the Heisenberg-like measures (radial expectation values of arbitrary orders) and the entropic measures of Fisher, Shannon and Rényi types. As well we have studied the corresponding uncertainty relations and the complexity measures of Crámer-Rao, Fisher-Shannon and LMC-Rényi types.

We have found a number of relevant results at first order of the pseudoclassical limit. Let us 
just highlight that (a) none of the hydrogenic and harmonic spreading quantities have any dependence on the state's hyperquantum numbers, (b) the hydrogenic and harmonic Heisenberg and entropic uncertainty relations based on Fisher, Shannon and Rényi entropies saturate the corresponding general uncertainty relations, and (c) the hydrogenic and harmonic Crámer-Rao and Fisher-Shannon complexities have the same values in the two conjugated spaces and attain their universal lower bounds. Then, neither the uncertainty relations nor these complexity measures are able to discern between the dominant forces of Coulomb and quadratic character which characterize the hydrogenic and harmonic systems, respectively. Finally, most interesting, the plain LMC and the generalized LMC-Rényi complexity measures are the only ones which can disentangle between the hydrogenic and harmonic systems already at first order in the high dimensional limit.

\section{Acknowledgments}

This work was partially supported by the grants FQM-7276 and FQM-207 of the Junta de Andalucía and the MINECO-FEDER (Ministerio de Economía y Competitividad, and the European Regional Development Fund) grants FIS2014-54497P and FIS2014-59311P. The work of I. V. Toranzo was financed by the program FPU of the Spanish Ministerio de Educación.

\section{References}

[1] Herschbach D R, Avery J and Goscinski O 1993 (Eds.) Dimensional Scaling in Chemical Physics ( London: Kluwer Acad. Publ.)

[2] Tsipis C T, Popov V S, Herschbach D R, Avery J S 1996 New Methods in Quantum Theory ( Kluwer Academic Publishers: Dordrecht, The Netherlands)

[3] Herschbach D R 1996 Int. J. Quant. Chem. 57295

[4] Herschbach D R 2000 Annu. Rev. Phys. Chem. 511

[5] Chatterjee A. 1990 Phys. Rep. 186249

[6] Svidzinsky A, Chen G, Chin S, Kim M, Ma D, Murawski R, Sergeev A, Scully M and Herschbach D 2008 Int. Rev. Phys. Chem. 27665

[7] Avery J 2000 Hyperspherical Harmonics and Generalized Sturmians (Kluwer, Dordrecht)

[8] Dong S H 2011 Wave Equations in Higher Dimensions (Springer, New York)

[9] Witten E 1980 Phys. Today 3338

[10] Yaffe L G 1982 Rev. Mod. Phys. 54407 
[11] Yaffe L G Phys. Today 19833650

[12] Weinberg S and Piran T 1986 (Eds.) Physics in Higher Dimensions (World Scientific Publi., Singapore)

[13] Kunstatter G 2003 Phys. Rev. Lett. 90161301

[14] Plenio M B, Hartley J, Eisert J 2004 New J. Phys. 2004636

[15] Rovenchak A 2014 Phys. Lett. A 2014378100

[16] Krenn M, Huber M, Fickler R, Lapkiewicz R, Ramelowa S and Zeilinger A 2014 PNAS 1116243

[17] Bellomo G, Plastino A R and Plastino A 2015 Int. J. Quantum Inf. 131550039

[18] Crann J, Kribs D W, Levene R H and Todorov I G 2016 J. Math. Phys. 57015208

[19] Ray A, Mahata K, Ray P P 1988 Am. J. Phys. 56462

[20] Angulo J C 1993 J. Phys. A 266493

[21] Angulo J C 2011 Phys. Rev. A 83062102

[22] Zozor S, Portesi M, Sánchez-Moreno P, Dehesa J S 2011 Phys. Rev. A 83052107

[23] Guerrero A, Sánchez-Moreno P and Dehesa J S 2011 Phys. Rev. A 84042105

[24] I. V. Toranzo, A. Martínez-Finkelshtein, and J. S. Dehesa, Heisenberg-like uncertainty measures for D-dimensional hydrogenic systems at large D. J. Math. Phys. 57, 082109 (2016).

[25] Al-Jaber S M 2016 Appl. Math. 7508

[26] Yáñez R J, Van Assche W, Dehesa J S 1994 Phys. Rev. A 503065

[27] Van Assche W, Yáñez R J, Dehesa J S 1995 J. Math. Phys. 364106

[28] Majernik V, Opatrny T 1996 J. Phys. A Math. Gen. 292187

[29] Dehesa J S, Yáñez R J, Aptekarev A I, Buyarov V 1998 J. Math. Phys. 393050

[30] Ghosh A, Chaudhuri P 2000 Int. J. Theor. Phys. 392423

[31] Dehesa J S, Martínez-Finkelshtein A, Sánchez-Ruiz J 2001 J. Comput. Appl. Math. 13323

[32] Sánchez-Moreno P, González-Férez R, Dehesa J S 2006 New J. Phys. 8330

[33] Dehesa J S, López-Rosa S, Martínez-Finkelshtein A, Yáñez R J 2010 Int. J. Quant. Chem. 1101529

[34] Aptekarev A I, Tulyakov D N, Toranzo I V, Dehesa J S 2016 Eur. Phys. J. B 8985

[35] Puertas-Centeno D, Toranzo I V, Dehesa J S 2017 Entropy 19164

[36] Puertas-Centeno D, Temme N M, Toranzo I V and Dehesa J S 2017 J. Math. Phys. Submitted

[37] Parr R G and Yang W 1989 Density-Functional Theory of Atoms and Molecules (Oxford University Press, Oxford)

[38] Akhiezer N I 1965 The Classical Moment Problem (Oliver and Boyd, London)

[39] Romera E, Angulo J C and Dehesa J S 2001 J. Math. Phys. 42 2309. Erratum 2003441

[40] Gadre S R and Pathak R K 1991 Adv. Quantum Chem. 22211

[41] Thakkar A J 2004 Adv. Chem. Phys. 128303

[42] Dehesa J S, Lopez-Rosa S and Manzano D 2012 In K.D. Sen (ed.) Statistical Complexities: Application to Electronic Structure (Springer, Berlin)

[43] Shannon C E 1948 Bell Syst. Tech. J. 27379

[44] Gyftopoulos E P and Cubukcu E 1997 Phys. Rev. E 553851 
[45] Onicescu O 1966 C.R. Acad. Sc. Paris Ser. A 263841

[46] Rényi A 1961 in Proc. Fourth Berkeley Symp. Math. Stat. Probability vol 1, p 547, reprinted in P. Durán 1973 (editor). Selected Papers of Alfred Rényi (Akademia Kiado, Budapest) p 565

[47] Leonenko N, Pronzato L, Savani V 2008 Ann. Stat. 402153

[48] Portesi M and Plastino A 1996 Physica A 225412

[49] Jizba P, Ma Y, Hayes A, Dunningham J A 2016 Phys. Rev. E 93060104

[50] Angulo J C, Antolin J and Esquivel R O 2012 In K.D. Sen (ed.) Statistical Complexities: Application to Electronic Structure (Springer, Berlin)

[51] Bialynicki-Birula I and Rudnicki L 2012 In K. D. Sen (ed.) Statistical Complexity: Applications in Electronic Structure (Springer, Berlin)

[52] Frieden B R 2004 Science from Frieden Information (Cambridge University Press)

[53] Luo S 2002 J. Phys. A: Math. Gen. 355181

[54] Romera E and Dehesa J S 1994 Phys. Rev. A 50256

[55] Nagy A and Romera E 2012 Phys. A 3913650

[56] Kinstner W 2009. A unified approach to fractal dimensions. See www.igi-global.com/chapter/unifiedapproach-fractal- dimensions $/ 27316$ ? camid $=4 \mathrm{v} 1$.

[57] Lau H K, Pooser R, Siopsis G and Weedbrook C 2017 Phys Rev Lett. 118080501

[58] González-Férez R and Dehesa J S 2003 Phys. Rev. Lett. 91113001

[59] González-Férez R and Dehesa J S 2005 Eur. Phys. J. D 3239

[60] Hall M J W 1999 Phys. Rev. A 592602

[61] Dembo A, Cover T M and Thomas J A 1991 IEEE Trans. Information Theor. 371501

[62] Sánchez-Moreno P, Zozor S and Dehesa J S 2011 J. Math. Phys. 52022105

[63] Sánchez-Moreno P, Plastino A R and Dehesa J S 2011 J. Phys. A: Math. Theor. 44065301

[64] Zozor S, Portesi M and Vignat C 2008 Physica A 3874800

[65] Dehesa J S, Sánchez-Moreno P, Yáñez R J 2006 J. Comput. Appl. Math. 186523

[66] Antolín J, Angulo J C 2009 Int. J. Quant. Chem. 109586

[67] Angulo J C and Antolín J 2008 J. Chem. Phys. 128164109

[68] Vignat C, Bercher J-F 2003 Physics Letters A 31227

[69] Angulo J C, Antolín J, Sen K D 2008 Phys. Lett. A 372670

[70] Romera E, Dehesa J S 2004 J. Chem. Phys. 1208906

[71] López-Ruiz R, Nagy Á, Romera E, Sañudo J 2009 J. Math. Phys. 50123528

[72] López-Ruiz R 2005 Biophys. Chem. 115215

[73] Pipek J, Varga I 1997 Int. J. Quant. Chem. 6485

[74] Romera E, Nagy A 2008 Physics Letters A 3726823

[75] Catalán R G, Garay J, López-Ruiz R 2002 Phys. Rev. E 66011102

[76] Yamano T 2004 J. Math. Phys. 451974

[77] Yamano T 2004 Physica A 340131 
[78] López-Rosa S, Angulo J C and Antolin J 2009 Physica A 3882081

[79] Olver F W J, Lozier D W, Boisvert R F and Clark C W 2010 NIST Handbook of Mathematical Functions (Cambridge: Cambridge University Press)

[80] Hey J D 1993 Am. J. Phys. 6128

[81] van Assche W, Yáñez R J, González-Férez R and Dehesa J S 2000 J.Math.Phys. 416600

[82] Temme N M, Toranzo I V and Dehesa J S 2017 J. Phys. A: Math. Theor. 50215206

[83] Romera E, Sánchez-Moreno P and Dehesa J S 2005 Chem. Phys. Lett. 414468

[84] Sánchez-Moreno P, González-Férez R and Dehesa J S 2006 New J. Phys. 8330 